\title{
Colouring the rational quantum sphere and the Kochen-Specker theorem
}

\author{
Hans Havlicek $^{1}$, Günther Krenn ${ }^{2}$, Johann Summhammer ${ }^{2}$ and Karl \\ Svozil $^{3,4}$ \\ ${ }^{1}$ Institut für Geometrie, Technische Universität Wien, Wiedner Hauptstraße 8-10/1133, A-1040 \\ Vienna, Austria \\ 2 Atominstitut der Österreichischen Universitäten, Stadionallee 2, A-1020 Vienna, Austria \\ ${ }^{3}$ Institut für Theoretische Physik, Technische Universität Wien, Wiedner Hauptstraße 8-10/136, \\ A-1040 Vienna, Austria \\ E-mail: havlicek@geometrie.tuwien.ac.at, krenn@ati.ac.at, summhammer@ati.ac.at and \\ svozil@tuwien.ac.at
}

Received 25 October 2000, in final form 26 February 2001

\begin{abstract}
We review and extend recent findings of Godsil and Zaks, who published a constructive colouring of the rational unit sphere with the property that for any orthogonal tripod formed by rays extending from the origin of the points of the sphere, exactly one ray is red, one white and one black. They also showed that any consistent colouring of the real sphere requires an additional colour. We discuss some of the consequences for the Kochen-Specker theorem.
\end{abstract}

PACS numbers: 0365B, 0210B, 0210G

\section{Colourings}

In what follows we shall consider 'rational rays'. A 'rational ray' is the linear span of a non-zero vector of $\boldsymbol{Q}^{n} \subset \boldsymbol{R}^{n}$.

Let $p$ be a prime number. A colouring of the rational rays of $\boldsymbol{R}^{n}, n \geqslant 1$, using $p^{n-1}+p^{n-2}+\cdots+1$ colours can be constructed in a straightforward manner. We refer to [3-5] for the theoretical background of the following construction.

Each rational ray is the linear span of a vector $\left(x_{1}, x_{2}, \ldots, x_{n}\right) \in Z^{n}$, where $x_{1}, x_{2}, \ldots, x_{n}$ are coprime. Such a vector is unique up to a factor \pm 1 .

Next, let $\boldsymbol{Z}_{p}$ be the field of residue classes modulo $p$. The vector space $\boldsymbol{Z}_{p}^{n}$ has $p^{n}-1$ non-zero vectors; each ray through the origin of $\boldsymbol{Z}_{p}^{n}$ has $p-1$ non-zero vectors. So there are exactly $\left(p^{n}-1\right) /(p-1)=p^{n-1}+p^{n-2}+\cdots+1$ distinct rays through the origin which can be coloured with $p^{n-1}+p^{n-2}+\cdots+1$ distinct colours.

Finally, assign to the ray $\operatorname{Sp}\left(x_{1}, x_{2}, \ldots, x_{n}\right)$ ('Sp' denotes linear span) the colour of the ray of $\boldsymbol{Z}_{p}^{n}$ which is obtained by taking the modulus of the coprime integers $x_{1}, x_{2}, \ldots, x_{n}$ modulo $p$.

4 To whom correspondence should be directed. 


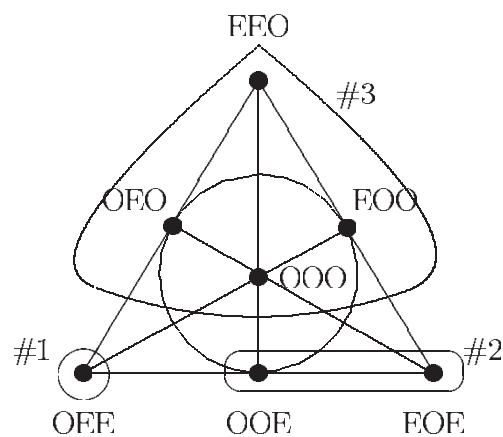

Figure 1. The projective plane over $Z_{2}$ and the reduced colouring scheme discussed.

Observe that $x_{1}, x_{2}, \ldots, x_{n}$ cannot vanish simultaneously modulo $p$ and that $\pm\left(x_{1}, x_{2}, \ldots, x_{n}\right)$ yield the same colour. Obviously, all $p^{n-1}+p^{n-2}+\cdots+1$ colours are actually used.

In what follows, we consider the case $p=2, n=3$. Here all rational rays $\operatorname{Sp}(x, y, z)$ (with $x, y, z \in Z$ coprime) are coloured according to the property of which of the components $x, y, z$ are even $(\mathrm{E})$ and odd $(\mathrm{O})$. There are exactly seven of such triples OEE, EOE, EEO, OOE, EOO, OEO, OOO which are associated with one of seven different colours \#1, \#2, \#3, \#4, \#5, \#6, \#7. Only the EEE triple is excluded. Those seven colours can be identified with the seven points of the projective plane over $Z_{2}$; cf figure 1 .

Next, we restrict our attention to those rays which meet the rational unit sphere $S^{2} \cap Q^{3}$. The following statements on a triple $(x, y, z) \in Z^{3} \backslash\{(0,0,0)\}$ (not necessarily coprime) are equivalent:

(i) The ray $\operatorname{Sp}(x, y, z)$ intersects the unit sphere at two rational points; i.e. it contains the rational points $\pm(x, y, z) / \sqrt{x^{2}+y^{2}+z^{2}} \in S^{2} \cap Q^{3}$.

(ii) The Pythagorean property holds, i.e., $x^{2}+y^{2}+z^{2}=n^{2}, n \in N$.

This equivalence can be demonstrated as follows. All points on the rational unit sphere can be written as $\boldsymbol{r}=\left(\frac{a}{a^{\prime}}, \frac{b}{b^{\prime}}, \frac{c}{c^{\prime}}\right)$ with $a, b, c \in Z, a^{\prime}, b^{\prime}, c^{\prime} \in Z \backslash\{0\}$, and $\left(\frac{a}{a^{\prime}}\right)^{2}+\left(\frac{b}{b^{\prime}}\right)^{2}+\left(\frac{c}{c^{\prime}}\right)^{2}=1$. Multiplication of $\boldsymbol{r}$ with $a^{\prime 2} b^{\prime 2} c^{\prime 2}$ results in a vector of $Z^{3}$ satisfying (ii). Conversely, from $x^{2}+y^{2}+z^{2}=n^{2}, n \in N$, we obtain the rational unit vector $\left(\frac{x}{n}, \frac{y}{n}, \frac{z}{n}\right) \in S^{2} \cap Q^{3}$.

Notice that this Pythagorean property is rather restrictive. Not all rational rays intersect the rational unit sphere. For a proof, consider $\operatorname{Sp}(1,1,0)$ which intersects the unit sphere at $\pm(1 / \sqrt{2})(1,1,0) \notin S^{2} \cap Q^{3}$. Although both the set of rational rays as well as $S^{2} \cap \boldsymbol{Q}^{3}$ are dense, there are 'many' rational rays which do not have the Pythagorean property.

If $x, y, z$ are chosen coprime then a necessary condition for $x^{2}+y^{2}+z^{2}$ being a non-zero square is that precisely one of $x, y$ and $z$ is odd. This is a direct consequence of the observation that any square is congruent to 0 or 1 , modulo 4 , and from the fact that at least one of $x, y$ and $z$ is odd. Hence our colouring of the rational rays induces the following colouring of the rational unit sphere with those three colours that are represented by the standard basis of $Z_{2}^{3}$ :

colour \#1 if $x$ is odd, $y$ and $z$ are even,

colour \#2 if $y$ is odd, $z$ and $x$ are even,

colour \#3 if $z$ is odd, $x$ and $y$ are even.

All three colours occur, since the vectors of the standard basis of $\boldsymbol{R}^{3}$ are coloured differently.

Suppose that two points of $S^{2} \cap Q^{3}$ are on rays $\operatorname{Sp}(x, y, z)$ and $\operatorname{Sp}\left(x^{\prime}, y^{\prime}, z^{\prime}\right)$, each with coprime entries. The inner product $x x^{\prime}+y y^{\prime}+z z^{\prime}$ is even if and only if the inner product of the corresponding basis vectors of $Z_{2}^{3}$ is zero or, in other words, the points are coloured differently. 
In particular, three points of $S^{2} \cap Q^{3}$ with mutually orthogonal position vectors are coloured differently.

From our considerations above, three colours are sufficient to obtain a colouring of the rational unit sphere $S^{2} \cap Q^{3}$ such that points with orthogonal position vectors are coloured differently, but clearly this cannot be accomplished with two colours. So the "chromatic number' for the rational unit sphere is three. This result is due to Godsil and Zaks [1]; they also showed that the chromatic number of the real unit sphere is four. However, they obtained their result in a slightly different way. Following [4] all rational rays are associated with three colours by making the following identification:

$$
\begin{aligned}
& \# 1 \\
& \# 2=\# 4 \\
& \# 3=\# 5=\# 6=\# 7 .
\end{aligned}
$$

This three-colouring has the property that coplanar rays are always coloured by using only two colours; cf figure 1. According to our approach this intermediate 3-colouring is not necessary, since rays in colours \#4, \#5, \#6, \#7 do not meet the rational unit sphere.

\subsection{Reduced two-colouring}

As a corollary, the rational unit sphere can be coloured by two colours such that, for any arbitrary orthogonal tripod spanned by rays through its origin, one vector is coloured by colour $\# 1$ and the other rays are coloured by colour \#2. This can be easily verified by identifying colours \#2 \& \#3 from the above scheme. (Two equivalent two-colouring schemes result from a reduced chromatic three-colouring scheme by requiring that colour $\# 1$ is associated with $x$ or $y$ being odd, respectively.)

Kent [6] has shown that there also exist dense sets in higher dimensions which permit a reduced two-colouring. Unpublished results by Ovchinnikov, Okunev and Mushtari [7] state that the rational $d$-dimensional unit sphere is d-colourable if and only if it admits a reduced two-colouring if and only if $d<6$.

\subsection{Denseness of single colours}

It can also be shown that each colour class in the above colouring schemes is dense in the sphere. To prove this, Godsil and Zaks consider $\alpha$ such that $\sin \alpha=\frac{3}{5}$ and thus $\cos \alpha=\frac{4}{5}$. $\alpha$ is not a rational multiple of $\pi$; hence $\sin (n \alpha)$ and $\cos (n \alpha)$ are non-zero for all integers $n$. Let $F$ be the rotation matrix about the $z$-axis through an angle $\alpha$; i.e.,

$$
F=\left(\begin{array}{ccc}
\cos \alpha & \sin \alpha & 0 \\
-\sin \alpha & \cos \alpha & 0 \\
0 & 0 & 1
\end{array}\right)
$$

Then the image $I$, under the powers of $F$, of the point $(1,0,0)$ is a dense subset of the equator.

Now suppose that the point $u=\left(\frac{a}{c}, \frac{b}{c}, 0\right)$ is on the rational unit sphere and that $a, c$ are odd and thus $b$ is even. In the colouring scheme introduced above, $u$ has the same colour as $(1,0,0)$ (identify $a=c=1$ and $b=0)$; and so does $F u$. This proves that $I$ (the image of all powers of $F$ of the points $u$ ) is dense. We shall come back to the physical consequences of this property later.

In the reduced two-colour setting, if the two 'poles' $\pm(0,0,1)$ acquire colour \#1, then the entire equator acquires colour \#2. Thus, for example, for the two tripods spanned by $\{(1,0,0),(0,1,0),(0,0,1)\}$ and $\{(3,4,0),(-4,3,0),(0,0,1)\}$, the first two legs have colour $\# 2$, while $(0,0,1)$ has colour \#1. 


\subsection{The chromatic number of the real unit sphere in three dimensions is four}

A proof that four colours suffice for the colouring of points of the unit sphere in three dimensions is constructive and rather elementary. Consider first the intersection points of the sphere with the $x$-, $y$ - and $z$-axes, coloured by green, blue and red, respectively. There are exactly three great circles which pass through two of these three pairs of points. The great circles can be coloured with the two colours used on the four points they pass through. The three great circles divide the sphere into eight open octants of equal area. Four octants, say, in the half space $z>0$, are coloured by the four colours red, white, green and blue. The remaining octants obtain their colour from their antipodal octant.

Although Godsil and Zaks [1] paper is not entirely specific, it is easy to write down an explicit colouring scheme according to the above prescription. Consider spherical coordinates: let $\theta$ be the angle between the $z$-axis and the line connecting the origin and the point, and $\varphi$ be the angle between the $x$-axis and the projection of the line connecting the origin and the point onto the $x-y$-plane. In terms of these coordinates, an arbitrary point on the unit sphere is given by $(\theta, \varphi, r=1) \equiv(\theta, \varphi)$.

- The colours of the cartesian coordinate axes $(\pi / 2,0),(\pi / 2, \pi / 2),(0,0)$ are green, blue and red, respectively.

- The colour of the octant $\{(\theta, \varphi) \mid 0<\theta \leqslant \pi / 2,0 \leqslant \varphi<\pi / 2\}$ is green.

- The colour of the octant $\{(\theta, \varphi) \mid 0 \leqslant \theta<\pi / 2, \pi / 2 \leqslant \varphi \leqslant \pi\}$ is red.

- The colour of the octant $\{(\theta, \varphi) \mid 0<\theta<\pi / 2, \pi<\varphi<3 \pi / 2\}$ is white.

- The colour of the octant $\{(\theta, \varphi) \mid 0<\theta \leqslant \pi / 2,-\pi / 2 \leqslant \varphi<0\}$ is blue.

- The colours of the points in the half space $z<0$ are inherited from their antipodes. This completes the colouring of the sphere.

The fact that three colours are not sufficient is not so obvious. Here we shall not review the proof of Godsil and Zaks based on a paper by Hales and Straus [4], but refer to a result of Kochen and Specker [2], which is of great importance in the present debate on hidden parameters in quantum mechanics. They have proven that there does not exist a reduced twocolouring, also termed valuation, on the one-dimensional subspaces of real Hilbert space in three dimensions.

Recall that a reduced two-colouring of the one-dimensional linear subspaces with two colours could immediately be obtained from any possible appropriate colouring of the sphere with three colours by just identifying two of the three colours. Thus, the impossibility of a reduced two-colouring implies that three colours are not sufficient for an appropriate colouring of the three-dimensional real unit sphere. ('Appropriate' here means: 'points at spherical distance $\pi / 2$ get different colours'.)

In the same article [2], Kochen and Specker gave an explicit example (their $\Gamma_{3}$ ) of a finite point set of the sphere with weaker properties which suffice just as well for this purpose: the structure still allows for a reduced two-colouring, yet it cannot be coloured by three colours. (The authors did not mention nor discuss this particular feature [9].)

The impossibility of a reduced two-colouring also rules out another attempt to 'nullify' the Kochen-Specker theorem by identifying pairs of colours of an appropriate four-colouring of the real unit sphere. Any such identification would result in tripods coloured by \#1-\#2-\#2, as well as for instance \#1-\#1-\#2, which is not allowed for reduced colouring schemes, which requires colourings of the type \#1-\#2-\#2. 


\section{Physical aspects}

\subsection{Physical truth values}

Based on the results of Godsil and Zaks [1], Meyer [10] suggested that the physical impact of the Kochen-Specker theorem [2] is 'nullified,' since for all practical purposes it is impossible to operationalize the difference between any dense set of rays and the continuum of Hilbert space rays (see also the subsequent papers by Kent [6] and Clifton and Kent [11]). However, for the reasons given below, the physical applicability of these constructions remain questionable.

Let us re-state the physical interpretation of the colouring schemes discussed above. Any linear subspace $\operatorname{Sp} \boldsymbol{r}$ of a vector $\boldsymbol{r}$ can be identified with the associated projection operator $E_{\boldsymbol{r}}$ and with the quantum mechanical proposition 'the physical system is in a pure state $E_{r}$ ' [12]. The colouring of the associated point on the unit sphere (if it exists) is equivalent with a valuation or two-valued probability measure

$$
\operatorname{Pr}: E_{r} \mapsto\{0,1\}
$$

where $0 \sim \# 2$ and $1 \sim \# 1$. That is, the two colours \#1, \#2 can be identified with the classical truth values: 'It is true that the physical system is in a pure state $E_{r}$ ' and 'It is false that the physical system is in a pure state $E_{r}$,' respectively.

Since, as has been argued before, the rational unit sphere has chromatic number three, two colours suffice for a reduced colouring generated under the assumption that the colours of two rays in any orthogonal tripod are identical. This effectively generates consistent valuations associated with the dense subset of physical properties corresponding to the rational unit sphere [10].

Kent [6] has shown that there also exist dense sets in higher dimensions which permit a reduced two-colouring. Unpublished results by Ovchinnikov, Okunev and Mushtari [7] state that the rational unit sphere of the $d$-dimensional real Hilbert space is d-colourable if and only if it admits a reduced two-colouring if and only if $d<6$.

\subsection{Sufficiency}

The Kochen-Specker theorem deals with the nonembedability of certain partial algebras-in particular the ones arising in the context of quantum mechanics - into total Boolean algebras. $0-1$ colourings serve as an important method of realizing such embeddings: e.g., if there are a sufficient number of them to separate any two elements of the partial algebra, then embedability follows [2,13,14]. Kochen and Specker's original paper [2] contains a much stronger resultthe nonexistence of $0-1$ colourings - than would be needed for nonembedability. However, the mere existence of some homomorphisms is a necessary but insufficient criterion for embedability. The Godsil and Zaks construction merely provides three homomorphisms which are not sufficient to guarantee embedability. This has already be pointed out by Clifton and Kent [11].

\subsection{Continuity}

The regress to unsharp measurements is rather questionable and would allow total arbitrariness in the choice of approximation. That is, due to the density of the colouring, depending on which approximation is chosen, one predicts different results. The probabilities resulting from these 
truth assignments are noncontinuous and arbitrary. This is even more problematic if one realizes that the each colour of the $0-1$ colouring of the rational unit sphere is dense.

\subsection{Closedness}

The rational unit sphere is not closed under certain geometrical operations such as taking an orthogonal ray of the subspace spanned by two non-collinear rays (the cross product of the associated vectors). This can be easily demonstrated by considering the two vectors

$$
\left(\frac{3}{5}, \frac{4}{5}, 0\right) \quad\left(0, \frac{4}{5}, \frac{3}{5}\right) \in S^{2} \cap Q^{3} .
$$

The cross product thereof is

$$
\left(\frac{12}{25}, \frac{-9}{25}, \frac{12}{25}\right) \notin S^{2} \cap Q^{3} .
$$

Indeed, if instead of $S^{2} \cap Q^{3}$ one would start with three non-orthogonal, non-collinear rational rays and generate new ones by the cross product, one would end up with all rational rays [15].

In the Birkhoff-von Neumann approach to quantum logics [16], this non-closedness under elementary operations such as the nor-operation might be considered a serious deficiency which rules out the above model as an alternative candidate for Hilbert space quantum mechanics. (However, his argument does not apply to the Kochen-Specker partial algebra approach to quantum logic, since there operations among propositions are only allowed if the propositions are comeasurable.)

Informally speaking, the relative (with respect to other sets such as the rational rays) 'thinness' guarantees colourability. In such a case, the formation of finite cycles such as the ones introduced by Kochen and Specker [2] are impossible.

To put it differently: given any nonzero measurement uncertainty $\varepsilon$ and any non-colourable Kochen-Specker graph $\Gamma(0)[2,17]$, there exists another graph (in fact, a denumerable infinity thereof) $\Gamma(\delta)$ which lies inside the range of measurement uncertainty $\delta \leqslant \varepsilon$ (and thus cannot be discriminated from the non-colourable $\Gamma(0)$ ) which can be coloured. Such a graph, however, might not be connected in the sense that the associated subspaces can be cyclically rotated into itself by local transformation along single axes. The set $\Gamma(\delta)$ might thus correspond to a collection of tripods such that none of the axes coincides with any other, although all of those non-identical single axes are located within $\delta$ apart from each other. Indeed, this appears to be precisely how the Clifton-Kent construction works [11, p 2104].

The reason why a Kochen-Specker-type contradiction does not occur in such a scenario is the impossibility to 'close' the argument; to complete the cycle: the necessary propositions are simply not available in the rational sphere model. For the same reason, an equilateral triangle does not exist in $Q^{2}$ [9]. Yet, while these findings seem to contradict the conclusions of Clifton and Kent [11], we would like to emphasize that this does not relate to their formal arguments but rather is a matter of interpretation and a question of how much should be sacrificed for value definiteness.

Thus, although the colourings of rational spheres offer a rather unexpected possibility to define consistent classical models, a closer examination shows that any such colourings should be excluded for physical reasons.

\section{Acknowledgment}

The authors would like to acknowledge stimulating discussions with Ernst Specker. 


\section{References}

[1] Godsil C D and Zaks J 1988 Colouring the sphere University of Waterloo Research Report CORR 88-12

[2] Kochen S and Specker E P 1967 The problem of hidden variables in quantum mechanics J. Math. Mech. 17 59-87 reprinted in [18, pp 235-63]

[3] Klingenberg W 1956 Projektive geometrien mit homomorphismus Math. Ann. 132 180-200

[4] Hales A W and Straus E G 1982 Projective colorings Pac. J. Math. 99 31-43

[5] Carter D S and Vogt A 1980 Collinearity-preserving functions between Desarguesian planes Mem. Am. Math. Soc. 23598

[6] Kent A 1999 Non-contextual hidden variables and physical measurements Phys. Rev. Lett. 83 3755-7 (Kent A 1999 Preprint arXiv:quant-ph/9906006)

[7] Ovchinnikov P 2000 Private communication

[8] Specker E 1960 Die Logik nicht gleichzeitig entscheidbarer Aussagen Dialectica $14175-82$ reprinted in [18, pp 175-82] (Engl. Transl. The Logic of Propositions which are not Simultaneously Decidable) reprinted in $[19$, pp 135-40]

[9] Specker E 1999 Private communication

[10] Meyer D A 1999 Finite precision measurement nullifies the Kochen-Specker theorem Phys. Rev. Lett. 83 $3751-4$ (Meyer D A 1999 Preprint arXiv:quant-ph/9905080)

[11] Clifton R and Kent A 2000 Simulating quantum mechanics by non-contextual hidden variables Proc. R. Soc. A 456 2101-14

(Clifton R and Kent A 1999 Preprint arXiv:quant-ph/9908031 v4)

[12] von Neumann J 1932 Mathematische Grundlagen der Quantenmechanik (Berlin: Springer) (Engl. Transl. 1955 Mathematical Foundations of Quantum Mechanics (Princeton, NJ: Princeton University Press))

[13] Pták P and Pulmannová S 1991 Orthomodular Structures as Quantum Logics (Dordrecht: Kluwer)

[14] Svozil K and Tkadlec J 1996 Greechie diagrams, nonexistence of measures in quantum logics and KochenSpecker type constructions J. Math. Phys. 37 5380-401

[15] Havlicek H and Svozil K 1996 Density conditions for quantum propositions J. Math. Phys. 37 5337-41

[16] Birkhoff G and von Neumann J 1936 The logic of quantum mechanics Ann. Math. 37 823-43

[17] Svozil K 1998 Quantum Logic (Singapore: Springer)

[18] Specker E 1990 Selecta (Basel: Birkhäuser)

[19] Hooker C A 1975-9 The Logico-Algebraic Approach to Quantum Mechanics (Boston: Reidel) 